\section{The PeriKit: an innovative connected portable device with high level of accuracy and reliability in taking circumferential limb measurements}

\section{Joseph Harfouche \\ Chirec Edith Cavell Clinics, Brussels, Belgium}

\section{Introduction}

Healthcare professionals, sportsmen and dieticians know how difficult it is to take circumferential limb measurements in a fast, reproducible and accurate way.

There is currently no device on the market able to take accurate and fast measurements and being affordable (size and price) at the same time.

For instance, in the field of lymphedema management, it is essential to reach the highest level of accuracy in the circumferential measurements in order to answer the healthcare insurances requirements, to follow-up the evolution of the edema, to evaluate and reevaluate the treatment but also to make sure that the compressive garments, (standard or made-to-measure) do fit perfectly the patients.

To be considered as accurate, scientists accept that the error can be of maximum a few millimeters.

The classical tapeline, which is the most frequently used measurement device, is affordable (size and price) but the measured values are approximate for multiple reasons like the perpendicularity of the tape, the pressure applied by the therapist, and the measurements are not taken each time exactly at the same place (unless we spend a lot of time drawing marks on the patients' skin, taking the risk to further stigmatize them and irritate or damage their sensitive skin).

Other devices, such as laser or infrared scanners, can be considered of high level of accuracy but they are very expensive and require space and an adapted infrastructure.

The water displacement which is considered as the gold standard is the reference in terms of accuracy. But the weaknesses are: price, time consuming, the water volume, the practicality, the hygiene. Since the whole volume is taken, there is no way to know where the change in volume took place on the limb.

The new perimeter measurement device and technic is called the PeriKit.
Taking measurements is made easy and fast thanks to its patented elements.

The Perikit consists in a graduated adaptable guide tapeline, installed longitudinally on the limb in order to: i) prevent marking the skin with the ink at the landmarks each $4 \mathrm{~cm}$, so it leaves no stigmas after each assessment and it protects the skin from scrubbing and possible damage when cleaning it; ii) to take the measurements on a non-elastic support and not on elastic and unstable skin; iii) to take the measurements faster; iv) to guarantee the exactitude of the place where the circumference are taken during the whole procedure.

On the guide tapeline, sliding devices (sld) are installed. On the first sld, a tapeline is fixed to take circumferential measurements and on the second sld, a brake system is fixed to stabilize the mechanism. Each sld is equipped with an aperture to read precisely the distance where the measurement is taken. To ensure its stability and accuracy on the limbs, the guide is fixed at its distal part to the joint (wrist or ankle) and will be stuck with bony landmarks (Figure 1 : unlar stiloïde for the upper limb; on the malleoli for the lower limb) that are more accurate than skin landmarks.

To check the reproducibility and accuracy concerning the reference point positioning and the circumferential measurements, the PeriKit has been tested on 43 healthy subjects who took part in a study. The choice of healthy subjects can guaranty that no bias of edema variability is possible and that any change in the measurement even of $1 \mathrm{~mm}$ is considered as a measurement inaccuracy and not a measurement variability. The measurements were taken on the upper limb each $4 \mathrm{~cm}$ beginning with

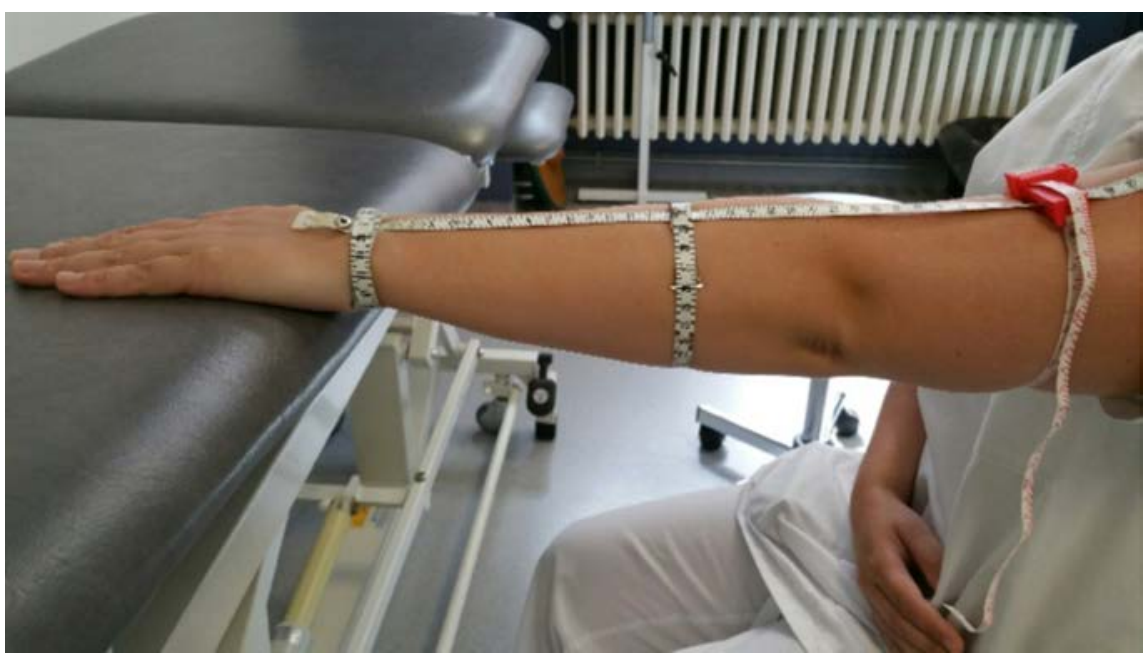

Figure 1. How to measure arm edema.
Correspondence: Joseph Harfouche, Chirec Edith Cavell Clinics, Brussels, Belgium. E-mail: joseph.harfouche@yahoo.com

This work is licensed under a Creative Commons Attribution 4.0 License (by-nc 4.0).

(C) Copyright J. Harfouche, 2017

Licensee PAGEPress, Italy

Veins and Lymphatics 2017; 6:6629

doi:10.4081/vl.2017.6629

a reference point on the wrist joint. The measurements were retaken independently within 1 hour.

Results: concerning the circumferential measurements, the interclass correlation (ICC) was 0.99. The Bland and Altman test confirmed the reproducibility of the system. Concerning the reference points taking/retaking, no statistically different changes were noticed between the first and the second assessment.

\section{Conclusions}

The PeriKit shows a high degree of reproducibility and accuracy with major advantages: i) $97 \%$ of the measurements taken showed a difference between 0 and 3 $\mathrm{mm}$; ii) high precision in retaking the bony landmark reference point that is fixed on the joint as well as the other landmarks and the mean difference was less than $2 \mathrm{~mm}$; iii) it solved the classic error of inaccuracy accumulations that can occur between each 4 $\mathrm{cm}$, because the PeriKit stays on the limb during the whole procedure; iv) no tension 
is put on the tapeline by the assessor, so the risk of affecting the accuracy is minimized; v) the PeriKit can adapt to any shape and length of any limb; vi) no ink marks on fragile skins are used, so the risk of abrasion followed by infection is solved.

A connected version of the PeriKit has also been developed.

This consists in a digital and connected PeriKit (IoT), linked to an app. The measurements are taken and instantly displayed simultaneously on the screen of the PeriKit as well as on any of the following devices where the app can be used: Laptops, Pads, smartphones, etc. The data can be compared in different charts and communicated to any authorized person (from the patient to the healthcare professionals)

This strengthens the features of the analogic version: i) faster measurements: the values are instantly displayed on the screen and in the app; ii) user-friendly: intuitive and easy to use and helps the assessor step by step; iii) faster treatment follow-up and adjustment: thanks to the charts and graphics that show the evolution of the edema at any distance and at any date.

As a conclusion, the PeriKit has been developed in order to facilitate the measurements taking and to improve the reliability of circumferential measurement data, especially in the field of lymphedema.

It is no longer, neither time consuming and laborious, nor a privilege for those who can afford it, to take reproducible and accurate measurements. Thanks to the PeriKit and its 2 versions (analogic and connected), almost anyone can easily reach a high level of reliability in taking measurements. 